\title{
Development of electrospun keratin/ coenzyme Q10/poly vinyl alcohol nanofibrous scaffold containing mupirocin as potential dressing for infected wounds
}

Joyce N. Amajuoyi ${ }^{1}$, Margaret O. Ilomuanya ${ }^{1,2^{*}}$ D, Yaa Asantewaa-Osei ${ }^{3}$, Chukwuemeka P. Azubuike ${ }^{1}$, Samson O. Adeosun ${ }^{4}$ and Cecilia I. Igwilo ${ }^{1}$

\begin{abstract}
Background: Nanostructured wound dressings produced by electrospinning biocompatible polymers possess great potential because they resemble the natural extracellular matrix and support cell adhesion, proliferation, and differentiation. This study seeks to fabricate mupirocin, keratin, and coenzyme Q10 (Co Q10)-loaded PVA electrospun scaffolds intended for wound healing application and to characterize their morphology, physical properties, antibacterial activity, and biocompatibility. Polyvinyl alcohol (PVA) (10\% w/v), various concentrations of keratin/Co Q10 fibrous scaffolds (electrospun at a voltage of $50 \mathrm{kV}$, flow rate of $4 \mathrm{~mL} / \mathrm{h}$ ), and $2 \%$ mupirocin was designed and fabricated to activate keratinocytes in the wound bed, stimulate cell proliferation, and increase antimicrobial penetration.

Results: The diameters of the scaffolds were observed to be in the nanoparticulate range $2.11 \pm 0.20$ to $3.27 \pm$ $0.10 \mathrm{~nm}$. By $30 \mathrm{~min}$, all the scaffolds had more than $50 \%$ of the cumulative concentration of mupirocin released with $51.06 \pm 2.104 \%$ to $74.66 \pm 1.72 \%$ of mupirocin released. At $1 \mathrm{~h}, 80 \%$ of the mupirocin in the scaffold was seen to have diffused out of the scaffold. Release of mupirocin was modulated; an initial burst release was followed by sustained release over 2 h. Electrospun keratin/Co Q10/PVA scaffold containing mupirocin showed excellent antimicrobial activity against all the clinical isolates of 2586, Staphylococcus aureus 2590, 2583, 2587, 2555. All the electrospun scaffolds showed higher cell viability values than the control at 48 and $72 \mathrm{~h}$, with the optimized CoQ10 scaffold concentration being $0.05 \% \mathrm{w} / \mathrm{w}$.

Conclusion: Electrospun nanofibers combining the biocompatibility potential of PVA with the bioactive nature of keratin $(0.01 \% \mathrm{w} / \mathrm{w})$ and CoQ10 $(0.5 \% \mathrm{w} / \mathrm{w})$ and the antibacterial property of mupirocin as a new potential for proper wound care was successfully developed. The cell line studies on this electrospun scaffold (PKCM 3) showed their ability to support the growth of keratinocytes hence the potential of developed scaffolds as a wound dressing. In vivo studies to further investigate the applications of the electrospun keratin/Co Q10/PVA nanofibrous scaffold as a wound dressing is however required.
\end{abstract}

Keywords: Wound dressing, Keratin, Coenzyme Q10, Polyvinyl alcohol, Mupirocin

\footnotetext{
*Correspondence: milomuanya@unilag.edu.ng; milomuanya@popcouncil.org

'Department of Pharmaceutics and Pharmaceutical Technology, Faculty of

Pharmacy, University of Lagos, PMB 12003, Surulere, Lagos, Nigeria

${ }^{2}$ Population Council, Center for Biomedical Research, Rockefeller University,

New York 10065, USA

Full list of author information is available at the end of the article
}

\section{Springer Open}

( ) The Author(s). 2020 Open Access This article is licensed under a Creative Commons Attribution 4.0 International License, which permits use, sharing, adaptation, distribution and reproduction in any medium or format, as long as you give appropriate credit to the original author(s) and the source, provide a link to the Creative Commons licence, and indicate if changes were made. The images or other third party material in this article are included in the article's Creative Commons licence, unless indicated otherwise in a credit line to the material. If material is not included in the article's Creative Commons licence and your intended use is not permitted by statutory regulation or exceeds the permitted use, you will need to obtain permission directly from the copyright holder. To view a copy of this licence, visit http://creativecommons.org/licenses/by/4.0/. 


\section{Background}

Chronic wound deviates from the normal sequence of physiologic interactions involved in wound healing [1, $2]$. It therefore is necessary to tailor interventions to match both causative pathophysiology and prevailing deficiencies at the wound site to promote healing [1]. Wound dressings play a vital role in the management of wounds through the protection of the wound from the external environment, the provision of moisture, and facilitation of the wound healing process [3]. Varying dosage forms for wound healing include antibacterial-based creams, ointments, and gauze dressings [3]. Antibacterial agents are incorporated into such wound dressings to lessen the risk of bacterial infection during healing process. To overcome the challenges and bridge the gap posed by the high cost of skin substitutes, wound management approaches which utilize nanoscale biomaterials are advocated. Nanoscale biomaterials have semblance to the extracellular matrix (ECM), both in structure and function, which affords them excellent capability to influence cellular pathways to tissue regeneration, through provision of conducive platforms for cell attachment, differentiation, and proliferation [4]. Electrospun fibers meet all these criteria hence their advantage as wound dressing materials. Electrospinning ensures that fibers possess high surface area to volume ratio necessary for large surface applications. Fiber functionalization and ease of materials combination is easily achieved when wound dressing materials are electrospun hence the advantage of electrospun fibers over traditional wound dressings fabricated via weaving fibers to obtained cotton or gauze wound dressings $[2,4]$.

A wound dressing material of desired functionality should possess a bio-inspired architecture that resembles the natural ECM organization in order to support cell adhesion, proliferation, and differentiation; the delivery of drugs and growth factors to accelerate tissue growth; and the controlled biodegradation of the scaffold to avoid further traumas during dressing detachment and peeling [4]. This is what thus makes electrospun fibers excellent wound dressing materials.

Polyvinyl alcohol (PVA) is a popular candidate for tissue engineering due to its hydrophilicity and biocompatibility which promote a conducive environment for skin regeneration. It however lacks adequate functional groups and cell recognition sites and to improve its cell affinity, it needs to be used in combination with bioactive composites to enhance cell adhesion and faster skin repair [5]. A good candidate for this purpose is keratin, due to its good bioactive potentials which support cell viability, cell attachment, and proliferation [6, 7]. Keratin has the ability to activate keratinocytes in the wound bed, stimulating them to proliferate and migrate leading to eventual wound closure [8].
Mupirocin is a bactericidal antimicrobial agent which has been used extensively to promote pathogen free wound bed [9]. It acts by competitive inhibitor of bacterial isoleucyl-tRNA synthetase, thereby impeding protein and RNA synthesis, which ultimately leads to bacterial death [9]. No systemic absorption of mupirocin or its major metabolite, monic acid, has been detected in short courses of topical administration and penetration into the deeper epidermal and dermal layers is enhanced in traumatized skin and under occlusive dressings [9].

Co-enzyme Q10 (CoQ10), also known as ubiquinone, is both a component in the electron transport chain and, when reduced to ubiquinol, it acts as an antioxidant. It substantially improves mitochondrial functions in diabetic wounds and plays an important role in diabetic wound by reducing the production of reactive oxygen species (ROS). This activity is very significant because of the ability ROS to disturb cellular signaling involved in wound healing, slowing the healing process [10]. Topical application of skin products containing Co Q10 can significantly increase the levels of ubiquinone on both the skin surface and deeper layers of the epidermis. Subsequent elevation of ubiquinol levels demonstrated after such topical therapy indicated metabolic transformation of ubiquinone. Incubation of cultured human keratinocytes with Co Q10 showed significant augmentation of energy metabolism [11].

This study seeks to fabricate mupirocin, keratin, and CoQ10-loaded PVA electrospun scaffolds intended for wound healing application and to characterize their morphology, physical properties, antibacterial activity, and biocompatibility. A novel nanofibrous co-spun mat containing keratin, mupirocin, and CoQ10 will offer greater chances of skin repair efficiency than any of the wound dressing materials currently in use for the management of chronic wounds.

\section{Method \\ Materials}

Mupirocin analytical standard (5,9-Anhydro-2,3,4,8-tetradeoxy-8-[[3-(2-hydroxy-1-methylpropyl)oxiranyl]methyl]-3-methyl-[2E,8[2S,3S(1S,2S)]]-L-talonon-2-enonic acid 8-carboxyoctyl ester, BRL 4910A, Pseudomonic acid. CAS number 12650-69-0 Sigma-Aldrich St. Louis, USA), mupirocin powder utilized as active pharmaceutical ingredient (CAS number 12650-69-0 Shaanxi Top Pharmaceutical Chemical Co. Limited, Shaanxi, China), polyvinyl alcohol (PVA with Degree of hydrolysis DH = 95; degree of polymerization $=1700$. Hebei Addtie Biotechnology Co. Limited China), keratin (Well-green Technology Co. Limited, Shaanxi, China). Acetic acid (Merck KGaA, Darmstadt, Germany), ethylene glycol diglycidyl ether (EGDE) from (Merck KGaA, Darmstadt, Germany). CoQ10 (Ubiquinone 50 CAS number 303-98- 
0 Sigma-Aldrich St. Louis, USA), phosphate buffer saline (PBS Merck KGaA, Darmstadt, Germany), Mueller-Hinton Agar (MHA-Merck KGaA, Darmstadt, Germany), Oxoid ${ }^{\mathrm{TM}}$ antimicrobial susceptibility disc for erythromycin and ciprofloxacin (ThermoFisher Scientific USA), resistant clinical isolates of Staphylococcus aureus 2586, Staphylococcus aureus 2590, Staphylococcus aureus 2583, Staphylococcus aureus 2587, methicillin-resistant Staphylococcus aureus 2555 (MRSA), and Escherichia coli 1808. Water used in all the tests was Milli-Q water (Millipore, USA). All other chemical reagents and solvents were of analytical grade and were used for this research without further purification.

\section{Electrospun scaffolds design and fabrication}

Using the method of Qiang et al. [5] with slight modification, five electrospinning solutions were each prepared by dissolving $10 \% \mathrm{w} / \mathrm{w}$ PVA in $18 \mathrm{~mL}$ of the distilled water/acetic acid solvent system. The mixture was transferred into a $200 \mathrm{~mL}$ conical flask and magnetically stirred at $300 \mathrm{rpm}$ at a temperature of $80{ }^{\circ} \mathrm{C}$ for $2 \mathrm{~h}$ and then reduced to $50{ }^{\circ} \mathrm{C}$ for $1 \mathrm{~h}$, followed by the addition of keratin dissolved in 10 $\mathrm{mL}$ of distilled water (Table 1). After $30 \mathrm{~min}$, mupirocin and/or Co Q10 (Table 1) were each separately dissolved in $2 \mathrm{~mL}$ of the water-acetic acid solvent and added to the PVA solution, followed by $0.1 \mathrm{~mL}$ of EGDE for cross linking of the polymer. The mixture was stirred at $300 \mathrm{rpm}$ for $30 \mathrm{~min}$ at $60{ }^{\circ} \mathrm{C}$ to ensure complete dissolution. The solutions were kept for 24 $\mathrm{h}$ to ensure homogeneity and then subjected to electrospinning. Electrospinning was carried out after process optimization under defined constant ambient condition (temperature: $25 \pm 1.56{ }^{\circ} \mathrm{C}$ and relative humidity $40 \%$ ). In each electrospinning solution, PKCM series (Table 1) was loaded into a $50 \mathrm{~mL}$ plastic syringe with an internal diameter of $0.8 \mathrm{~mm}$, length of $20 \mathrm{~mm}$, and fitted with a gauge 19G needle. The solutions were fed at $4.0 \mathrm{~mL} / \mathrm{h}$, and this was achieved by fall under gravity. The collector was placed at 15 $\mathrm{cm}$ from the needle tip to the collector. Electrospun fiber mats were collected by applying a voltage at 50 $\mathrm{kV}$ in the needle. The electrospun scaffolds were stored in airtight containers and suitable samples withdrawn intermittently for analysis.

\section{Mechanical characterization}

The mechanical strength, i.e., ultimate tensile strength (UTS) and ductility of the nanofibrous mats, was determined using Universal Tensile Machine (UTM Instron- series 3369, USA), fitted with $50 \mathrm{kN}$ load cell at $25 \pm 2{ }^{\circ} \mathrm{C}$. The UTS is a material's maximum resistance to fracture. It is equivalent to the maximum load that can be carried by one square inch of cross-sectional area when the load is applied as simple tension. Ductility measured the ability of a material to be drawn or plastically deformed without fracture.

The cross-linked scaffolds were cut into dog boneshaped sections $(50 \mathrm{~mm} \times 13.5 \mathrm{~mm})$ to control failure location and tested in ambient temperature of $20^{\circ} \mathrm{C}$ and humidity of $60 \%$. The sections of each of the electro spun mats (dimensions $13.5 \mathrm{~cm}$ ) were mounted between two clamps and stretched at rate of $50 \mathrm{~mm} / \mathrm{min}$ with an applied load range of $50 \mathrm{~N}$ and gauge length of $50 \mathrm{~mm}$ [12]. UTS and ductility determinations were done in triplicate.

\section{Morphological characterization \\ Scanning electron microscopy}

The surface morphology of the fabricated electrospun keratin/Co Q10/PVA nanofibrous scaffolds was determined using field emission scanning electron microscopy (FEG-SEM) via a Phenom World Eindhoven Phenom ProX scanning electron microscope at an accelerating voltage of $15 \mathrm{kV}$. The scaffolds were cut into small pieces of $5 \mathrm{~mm} \times 5 \mathrm{~mm}$. Before observation, each sample fibers were sputtered coated with thin layer of gold using a quorum-Q150R Plus E. The $5 \mathrm{~mm} \times 5 \mathrm{~mm}$ cut samples were then mounted over the studs using a carbon tape and analyzed at $15 \mathrm{KV}$. All the various samples were analyzed in triplicate.

\section{Moisture vapor transmission rate}

A $40 \mathrm{~mm}$ diameter sample of each electrospun scaffold of the series was fixed over a calibrated cylindrical vial (Smith Scientific glassware Edenbridge, Kent UK) of 35.7 $\mathrm{mm}$ inner diameter, containing $20 \mathrm{~mL}$ of distilled water. The test sample container was weighed (W1) before the start of the test and the container was then transferred to an incubator for $24 \mathrm{~h}$ (temperature $37.0 \pm 0.5{ }^{\circ} \mathrm{C}$ and $\mathrm{RH} 20 \%$ ). At the end of the test period, the container was reweighed (W2) to observe any changes in weight

Table 1 Composition of the solutions for electrospinning

\begin{tabular}{|c|c|c|c|c|c|}
\hline \multirow{2}{*}{$\begin{array}{l}\text { Electrospun } \\
\text { component }\end{array}$} & \multicolumn{5}{|c|}{ Electrospun PVA/keratin/Co Q10/Mupirocin scaffold formulation } \\
\hline & PKCM1 & PKCM2 & PKCM3 & PKCM4 & PKCM5 \\
\hline PVA (\% w/w) & 10 & 10 & 10 & 10 & 10 \\
\hline Keratin (\% w/w) & 0.01 & 0.1 & 0.01 & 0.1 & 0.05 \\
\hline CoQ10 (\% w/w) & 0.05 & - & 0.05 & 0.1 & 0.15 \\
\hline Mupirocin (\% w/w) & - & 2 & 2 & 2 & 2 \\
\hline
\end{tabular}


due to water absorption. The moisture vapor transmission rate (MVTR) was obtained from Eq. (1).

$$
X=(W 1-W 2) \times 1000 \times 24 / T
$$

where $X$ is MVTR $\left(\mathrm{g} / \mathrm{m}^{2} / 24 \mathrm{~h}\right) ; W 1$ is the initial mass of the container, sample, and liquid in grams, $W 2$ is the mass of the container, sample, and liquid in grams after the test duration; and $T$ is the test period in hours [3].

\section{Determination of the porosity of the electrospun scaffolds}

The porosity of the scaffolds was determined using the "liquid displacement method" [13] with ethanol used as the displacement liquid. The weight of the scaffold used was noted as "w." The scaffold was then immersed in 5 $\mathrm{mL}$ of ethanol and volume of ethanol (V1) was noted. After $5 \mathrm{~min}$, the volume of ethanol present in the ethanol-impregnated scaffold (V2) and the volume of ethanol after removal of the ethanol-impregnated scaffold (V3) was used to calculate porosity of the fiber as in Eq. (2). All measurements were achieved using a calibrated cylindrical vial (Smith Scientific glassware Edenbridge, Kent UK) and performed in triplicate.

$$
\text { Porosity of the fiber }=\frac{V 1-V 3}{V 2-V 3}
$$

\section{Chemical characterization $X$-ray diffraction}

The X-ray diffraction patterns of the electrospun inning fibers were obtained using an X-ray diffractometer (D/ MAX-2500X, Rigaku, Tokyo, Japan) with $\mathrm{Cu} \mathrm{K \alpha}$ characteristic radiation (wavelength $\lambda=0.154 \mathrm{~nm}$ at $40 \mathrm{kV}$, $150 \mathrm{~mA}$, and a scan speed of $4^{\circ}$ per minute in the $2 \theta$ range of $5^{\circ}-80^{\circ}$ ) [7].

\section{Fourier transform infrared spectroscopy}

The infra-red spectra of the electrospun scaffolds were obtained using a Fourier transform infrared spectrometer (Cary 630 FTIR Spectrometer; Agilent Technologies, Santa Clara, CA, USA). The spectral range was 4000 $\mathrm{cm}^{-1}$ to $400 \mathrm{~cm}^{-1}$. Then, $0.5 \mathrm{~g}$ of nanofibrous mats were dried in a vacuum oven $\left(45^{\circ} \mathrm{C}\right)$ and subsequently placed over the diamond crystal for analysis. Twenty scans were recorded for each spectrum. Smoothing was done where necessary to reduce the noise, without loss of any peak. The absorptions peaks/bands were studied to know if there was any chemical interaction occurring as a result of the co-formulation.

\section{In vitro release of mupirocin from PKCM scaffolds}

Using a modified in situ glass vial system, a patch of PKCM scaffold $(4 \mathrm{~cm} \times 4 \mathrm{~cm})$ was immersed into $50 \mathrm{~mL}$ of phosphate buffer solution ( $\mathrm{pH} 7.4)$. The samples were incubated at $37 \pm 0.5{ }^{\circ} \mathrm{C}$ and stirred at $100 \mathrm{rpm}$ using a magnetic stirrer. Aliquots of each sample $(0.5 \mathrm{~mL})$ were taken from the release medium at specific intervals $(0,5$, 15,30 , and $60,75 \mathrm{~min}$ ) and replaced with $0.5 \mathrm{~mL}$ with fresh buffer solution maintain sink condition [14]. The solution was subsequently filtered using nylon membrane of $0.45 \mu \mathrm{m}$ porosity. The resultant drug solution was analyzed by high-performance liquid chromatography using $\mathrm{C} 18$ column $(250 \times 4.6 \mathrm{~mm}, 5 \mu \mathrm{m})$ at $25^{\circ} \mathrm{C}$ and the mobile phase combination of ammonium acetate $(0.05 \mathrm{M}$ adjusted to $\mathrm{pH} 5.0$ with acetic acid) and acetonitrile $60: 40(\mathrm{v} / \mathrm{v})$. The injection volume was $20 \mu \mathrm{L}$ and the flow rate $1.0 \mathrm{~mL} / \mathrm{min}$; the ultraviolet detection was set at $228 \mathrm{~nm}$. The reported values are average of five readings \pm SD [14]. LOD release for mupirocin release was linear between 1 and $100 \mu \mathrm{g} / \mathrm{mL}\left(R^{2}=0.998\right)$.

\section{Biological characterization Skin irritation}

Twenty-four 3-month-old male and female rats with body weight $230-240 \mathrm{~g}$ were purchased form Tosab ${ }^{\circ}$ Laboratories Ogbomoso Osun State. They were individually housed and free access to standard chow and drinking water. They were allowed to acclimatize to the environment for 7 days prior to the start of the experiments and were maintained on a 12-h light/dark cycle. This study was carried out in strict accordance with the recommendations in the Guide for the Care and Use of Laboratory Animals of the National Institutes of Health $[15,16]$. This study utilized the Animal research: reporting in vivo experiments: the ARRIVE guidelines in documenting the study [16]. The protocol was approved by Health research ethics committee (CMUL/HREC/09/19/607) (Table 2).

The skin irritation test was performed on the electrospun PKCM nanofibers in healthy albino rats weighing averagely $238 \mathrm{~g} \pm 1.45 \mathrm{~g}$. The rats were randomly

\begin{tabular}{|c|c|c|}
\hline Reaction & Description & Score \\
\hline \multirow[t]{4}{*}{ Erythema } & No erythema & 3 \\
\hline & Slight erythema (not very noticeable) & 2 \\
\hline & $\begin{array}{l}\text { Moderate erythema (noticeable,but not covering the } \\
\text { entire test area) }\end{array}$ & 1 \\
\hline & $\begin{array}{l}\text { Severe erythema (noticeable and covering the entire } \\
\text { test area) }\end{array}$ & 0 \\
\hline \multirow[t]{4}{*}{ Edema } & No oedema (no swelling within the entire test area) & 3 \\
\hline & $\begin{array}{l}\text { Slight edema ( } 1 \text { swollen spot, having an area not } \\
\text { more than } 0.5 \mathrm{~mm}^{2} \text { ) }\end{array}$ & 2 \\
\hline & $\begin{array}{l}\text { Moderate edema } \\
\text { ( } 2-3 \text { swollen spots, each having an area not more } \\
\text { than } 0.5 \mathrm{~mm}^{2} \text { ) }\end{array}$ & 1 \\
\hline & Severe edema (up to half of the test area is swollen) & 0 \\
\hline
\end{tabular}

Table 2 Scale for assessing skin irritancy reactions 
divided into six groups (three rats in a group). Exposure of skin to the test materials was accomplished by means of a patch test technique using two intact sites on the back of each of the eighteen albino rats. The skin was clipped free of hair a day prior to testing. A square patch of the five electrospun scaffolds, with the dimension of $2.5 \mathrm{~cm} \times 2.5 \mathrm{~cm}$ were applied to the test site on the corresponding test group and covered with gauze of same dimension.

Mupirocin ointment was applied to group 6 and covered with gauze. PKCM1, PKCM2, PKCM3, PKCM4, and PKCM5 were tested for the presence of erythema or oedema. Group 1 rats served as the negative control group and the test areas of the skin were covered with polyethylene sleeve only. Group 6 rats were the positive control group and were treated with $2 \%$ mupirocin ointment. This was followed by the occlusion of the entire trunk using a polyethylene sleeve. The entire covering was removed after $24 \mathrm{~h}$ and the site assessed and scored for erythema and edema. Following the administration of the test samples and controls $(24,48$, or $72 \mathrm{~h})$. At the end of the study, all the animals were humanely euthanized via exposure to carbon dioxide gas in a sealed chamber.

\section{Antibacterial activity assessment}

This was done by disk diffusion method using Mueller-Hinton Agar (MHA) as the medium. Using sterile swaps, the bacterial culture suspensions of resistant clinical isolates of Staphylococcus aureus 2586, Staphylococcus aureus 2590, Staphylococcus aureus 2583, Staphylococcus aureus 2587, methicillin-resistant Staphylococcus aureus 2555 (MRSA), and Escherichia coli 1808 standardized to a turbidity of 0.5 McFarland standard were spread on different plates of MHA. In vitro antibacterial activity of PKCM electrospun nanofibrous scaffold against each bacterial strain was determined via disc diffusion test [14] using $150 \mathrm{~mm}$ diameter Mueller-Hinton (Carolina Biological Supply Co. Burlington, NC) agar plates. PKCM electrospun scaffolds were cut into $10 \mathrm{~mm}$ diameter and placed on the inoculated test organisms and then observed for any zone of inhibition around the scaffolds. Clear inhibition zones around the PCKM electrospun nanofibrous discs indicate that the scaffolds possess antimicrobial activity. Oxoid ${ }^{\mathrm{TM}}$ antimicrobial susceptibility disc for erythromycin and ciprofloxacin were used as control for antibacterial assessment. Each assay was performed in triplicate and incubated in an inverted position at $37 \pm 1{ }^{\circ} \mathrm{C}$ for $18 \mathrm{~h}$ and the zones of inhibition was measured. The protocol was approved by Health research ethics committee (CMUL/HREC/09/ 19/607).

\section{Cytotoxicity and cell proliferation assay}

The stock solutions were prepared by dissolving $26.5 \mathrm{mg}$ of each of the scaffolds in $4 \mathrm{~mL}$ of phosphate buffer $\mathrm{pH}$ 7.4. and sterilized by filtration using of $0.20 \mu \mathrm{m}$ pore size filter and subsequently stored in the refrigerator until needed for the tests. The CellTiter-Glo ${ }^{\circ} 2.0$ assay kit (Promega) was used to determine cell viability, as described by the manufacturer. Briefly, $1.0 \times 10^{4}$ keratinocyte ( $\mathrm{HaCaTs}$ ) cells were seeded in a luminometer compatible 96-well plate and allowed to grow and adhere overnight at $37{ }^{\circ} \mathrm{C}$ in a $5 \% \mathrm{CO}_{2}$ culture incubator. Treatments were performed by adding a 1:1 scaffold solution/media mix to the cells for $24 \mathrm{~h}$ and $72 \mathrm{~h}$ respectively. The plate and its contents were equilibrated to 25 $\pm 1{ }^{\circ} \mathrm{C}$ for approximately $30 \mathrm{~min}$ and then a volume of CellTiter-Glo 2.0 reagent equal to the volume of cell culture medium present in each well was added. The contents were subsequently mixed for 2 min using an orbital shaker to induce cell lysis and then followed by plate incubation at room temperature for $10 \mathrm{~min}$ to stabilize the luminescent signal and finally the luminescence was recorded using luminometer (MODULUS, Promega). The luminescent signal is proportional to the amount of ATP in the sample, which indicates the presence of metabolically active cells.

Cell viability is expressed in Eq. (3).

$$
\% \text { Cell viability }=\frac{\text { sample luminescence }}{\text { average luminescence } x} \times 100
$$

\section{Antioxidant activity of the of the PKCM scaffolds using DPPH assay}

The capacity of CoQ10 molecule (Ubiquinone) to act as free radical scavenging antioxidant after it has been incorporated in the electrospun scaffolds was determined using DPPH assay. Spectrophotometric determination of the free radical scavenging activity was evaluated using a method described by Ilomuanya et al. [17]. The radical scavenging activity of the scaffolds against 1,1 diphenyl11-picryl-1-hydrazyl (DPPH) radical via UV-Vis absorbance at $517 \mathrm{~nm}$ was determined utilizing ascorbic acid as standard. Further, $100 \mathrm{mg}$ of the electrospun scaffolds was weighed out and the antioxidant content extracted using $50 \mathrm{~mL}$ absolute ethanol in a separating funnel to obtain the test sample. To a methanolic solution of DPPH $(100 \mathrm{mmol} / \mathrm{L} 2 \mathrm{~mL})$, varying concentrations (5$25 \mathrm{mg} / \mathrm{mL}$ ) of the test sample was further dissolved in 3 $\mathrm{mL}$ ethanol and assayed. When DPPH reacts with an antioxidant compound, which can donate hydrogen, it is reduced. The changes in color (from deep violet to light yellow) after $100 \mathrm{~min}$ of reaction was determined using a UV-Visible Spectrophotometer (Unico S2150UV NJ, USA) with absorbance recorded at $517 \mathrm{~nm}$. A 
commercial antioxidant Citrix ${ }^{\circ}$ Topix Pharmaceuticals, Inc. NY, USA was also evaluated as antioxidant control and compared with the fabricated scaffolds. \% Scavenging activity was calculated using Eq. 4.

$\%$ Scavenging activity $=\frac{\text { Absorbance }_{517 \text { control }}-\text { Absorbance }_{517} \text { sample }}{\text { Absorbance }_{517 \text { control }}} \times 100$

\section{Statistical analysis}

The data was presented as mean \pm standard deviation of more than three experimental values for every variable and analyzed by one-way ANOVA followed by Dunnett's test using GraphPad Prism version 5. P value of less than 0.05 was considered statistically significant.

\section{Results}

Mechanical characterization

PKCM2 scaffold which did not contain CoQ10 had least tensile strength of $0.03 \mathrm{MPa}$, while PKCM4 containing $0.1 \%$ CoQ10 had the highest value of $0.20 \mathrm{MPa}$ (Fig. 1a, b). PKCM3 scaffold containing CoQ10 0.05\% had highest ductility value of $81.2 \%$, while PKCM4 containing $0.1 \%$ CoQ10 had the lowest value of $44.2 \%$. Variations in ductility of electrospun PKCM electrospun scaffolds are shown in Fig. 1b. Research has confirmed that a high elastic material will possess a high value of elongation at break [18]. PKCM3 scaffold showed the best capacity to deform before breakage with a ductility of $81.2 \%$. PKCM1 shows $78.7 \%$ response to deformation which is second to PKCM3. PKCM2, PKCM4, and PKCM5 reveal ductility values of $57.9 \%, 44.2 \%$, and $70.7 \%$. The reduced ductility values could be attributed to clustering of particles making each component to act as a rigid constituent which obstructs the mobility of crazes during tensile loading [18].

\section{Morphological characterization}

Scanning electron microscopy, moisture vapor transmission rate, and porosity of the electrospun scaffolds

All the electrospun scaffolds developed has sizes in the nanometer range. Different morphological features of

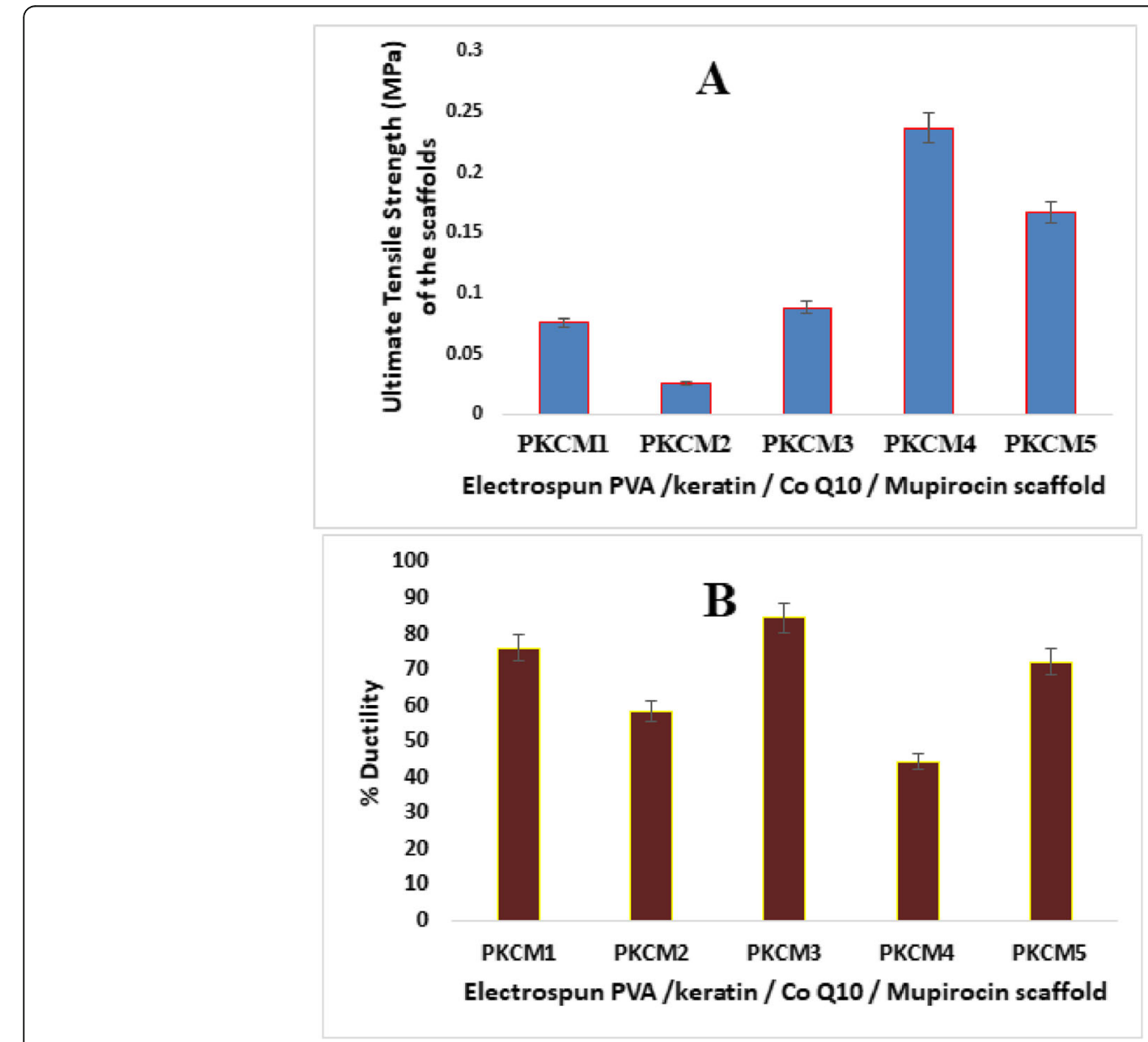

Fig. 1 a Ultimate tensile strength and (B)\% Ductility of the electrospun PVA/keratin Co Q10 mupirocin scaffolds 
electrospun fibers are shown in Fig. 2a. The diameters of the scaffolds were observed to be in the nano particulate range. The values were $3.27 \pm 0.1 \mathrm{~nm}, 2.61 \pm 0.3 \mathrm{~nm}$, $2.36 \pm 0.1 \mathrm{~nm}, 2.32 \pm 0.2 \mathrm{~nm}$, and $2.11 \pm 0.2 \mathrm{~nm}$ for PKCM1, PKCM2, PKCM3, PKCM4, and PKCM5 respectively. It is observed that fibers of sample PKCM1 (Fig. 2a) have varying diameters with few beads and relatively smooth surfaces. Fibers of sample PKCM2 (Fig. 2a) have near uniform diameter with a smooth surface while fibers of samples PKCM3, PKCM4, and PKCM5 have uniform densely knit scaffolds with near uniform diameters. The formation of uniform densely knit scaffolds could be attributed to surface tension of electrospinning solutions. Wound dressings should be able to protect the surface of the wound from drying out; healthy skin possess moisture vapor transmission rate
(MVTR) 700-1200 g/m² [19]. The developed PKCM scaffolds exhibited MVTR in the range $709 \pm 1.75-758$ $\pm 1.75 \mathrm{~g} / \mathrm{m}^{2}$ for PKCM1 and PKCM3. The highest MVTR was exhibited by PKCM 3, and PKCM 1 having the lowest values all within the desired limits to prevent excessive hydration of the wound bed. The porosity of the samples is $62.6 \pm 3.2 \%, 66 \pm 4.3 \%, 73.3 \pm 2.8,65.3 \pm$ $4.5 \%$, and $68.1 \pm 2.6 \%$ for PKCM1, PKCM2, PKCM3, PKCM4, and PKCM5 respectively. This shows that the difference in the concentration of the keratin loaded in the fibers may influence fiber porosity.

\section{Chemical characterization \\ $X$-ray diffraction}

Diffraction patterns of reinforced PKCM1, PKCM2, PKCM3, PKCM4, and PKCM5 scaffolds are shown in

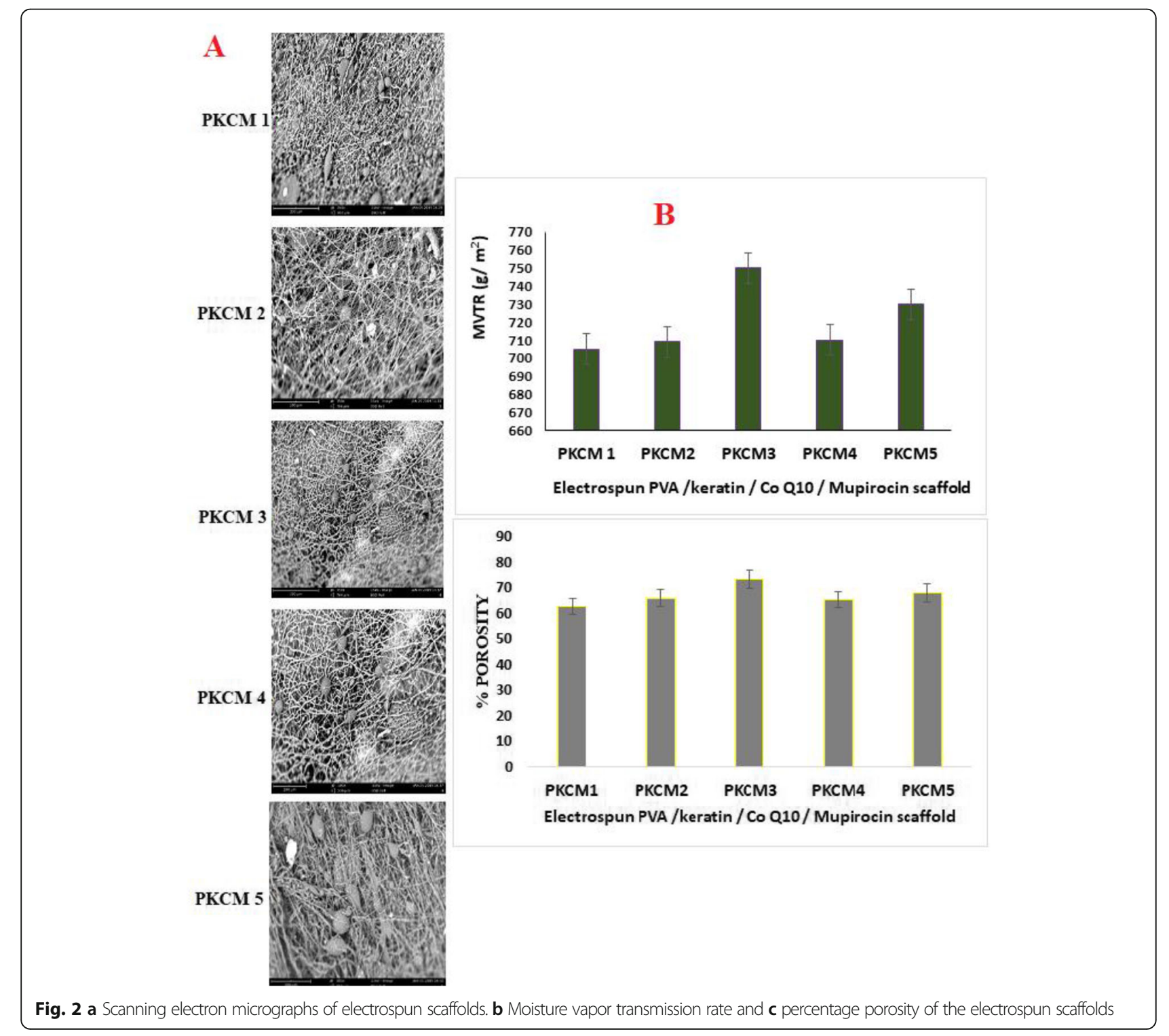


Fig. 3a. Two characteristic peaks existing at $2 \theta=19.7^{\circ}$ and $40.8^{\circ}$ are related to the semi-crystalline nature of PVA and as such, the scaffolds possess similar diffraction patterns. The variations in the additives used in the preparation of the precursor solutions for the scaffolds culminated in the formation of different peak intensities and sizes. PKCM4 possesses the highest intensity in the stronger diffraction angle $\left(2 \theta=19.7^{\circ}\right)$ with the narrowest peak, thus indicating the highest crystallinity. This means that the cross-linking agent performed optimally at the ratio of the additives used in the formulation of PKCM4. This also collaborates with the observation that PKCM4 scaffold possessed the greatest mechanical strength than the other scaffolds produced in this study. This is evident in the highest magnitude of tensile strength exhibited by this sample compared to other electrospun scaffolds (Fig. 1a).

\section{Fourier transform infrared spectroscopy}

Figure $3 \mathrm{~b}$ shows the Fourier transform infrared spectroscopy (FTIR) spectra in the region $4000-600 \mathrm{~cm}^{-1}$ of the scaffold formulation. FTIR shows that the large bands observed between 3500 and $3200 \mathrm{~cm}^{-1}$ are linked to the stretching $\mathrm{O}-\mathrm{H}$ from the intermolecular and intramolecular hydrogen bonds. Characteristic bands for $\alpha$-helix, $\beta$-sheet, and random coil conformations in the amide I $(\mathrm{C}=\mathrm{O}$ stretch vibrations of the peptide linkages 1700 $1600 \mathrm{~cm}^{-1}$ ) and amide II (N-H bending and from the C$\mathrm{N}$ stretching vibration $1560-1500 \mathrm{~cm}^{-1}$ ) regions [20] indicative of the presence of keratin. There is a 3000-2800 $\mathrm{cm}^{-1}$ vibration band due to stretching $\mathrm{C}-\mathrm{H}$ from alkyl groups, and $1750-1690 \mathrm{~cm}^{-1}$ peaks showing $\mathrm{C}=\mathrm{O}$ and
C-O stretching indicative of PVA. The FTIR spectra PKCM1 to 5 showed characteristic bands at $1712 \mathrm{~cm}^{-1}$ corresponding to $\mathrm{C}=\mathrm{O}$ stretching, at 2859 and 2929 $\mathrm{cm}^{-1}$ corresponding to $\mathrm{C}-\mathrm{H}$ deformation, at $1150 \mathrm{~cm}^{-1}$ corresponding to the $\mathrm{C}-\mathrm{O}-\mathrm{C}$ stretching vibration, and at $1052 \mathrm{~cm}^{-1}$ corresponding to the $\mathrm{C}-\mathrm{C}$ skeleton which are indicative of the presence of mupirocin [20]. The spectrum shows no indication of chemical interactions in all the PKCM spectra hence mupirocin and keratin were successfully incorporated into the scaffolds during electrospinning.

\section{In vitro release of mupirocin from PKCM scaffolds}

The ability of the scaffold to effectively modulate the release of mupirocin is critical to the success of the developed scaffold. The electrospun scaffolds must be seen to have the ability to release the active ingredient at the site of action. The drug release of mupirocin for the PKCM scaffolds was obtained at $r^{2}=0.998$ at wavelength of 228 $\mathrm{nm}$. There was an initial burst release of mupirocin from the scaffolds. PKCM 4 had $27.25 \pm 0.19 \%$ release at 5 min. At 30 min, all the scaffolds had more than $50 \%$ of the cumulative concentration of mupirocin released with $51.06 \pm 2.104 \%, 70.31 \pm 1.99 \%, 74.11 \pm 2.36 \%$, and 74.66 $\pm 1.72 \%$ of mupirocin released respectively for PKCM 2 , PKCM 3, PKCM 4, and PKCM 5. At $1 \mathrm{~h}, 80 \%$ of the mupirocin in the scaffold was seen to have diffused out of the scaffold (Fig. 4), hence buttressing the presence of reversible chemical linkages in the scaffolds that are not interfering with the release of the API mupirocin hence allowing the drug to be available at the site of action when it is applied on an actual wound.
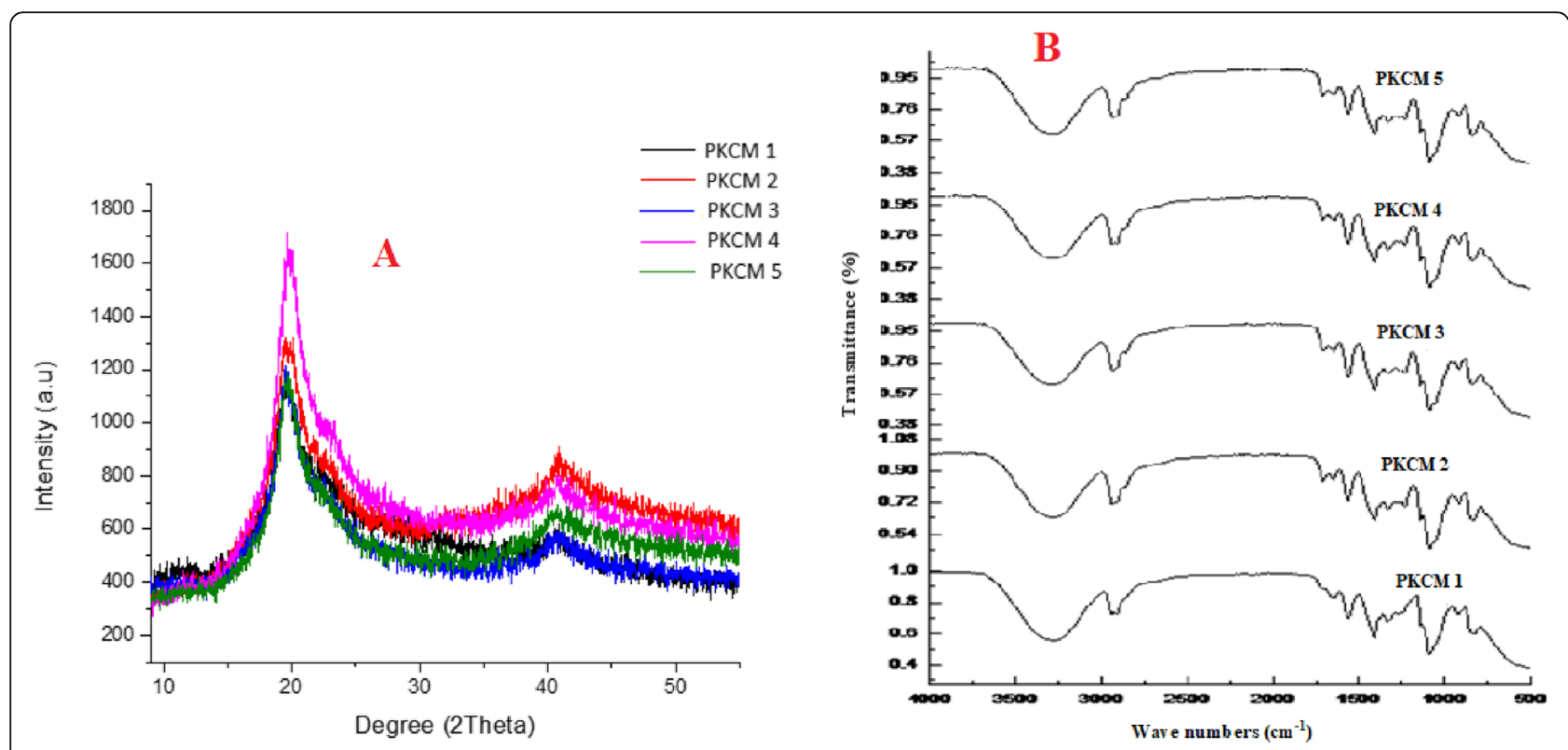

Fig. 3 a X-ray diffraction curves and $\mathbf{b}$ Fourier transform infrared spectra for PKCM1, PKCM2, PKCM3, PKCM4, and PKCM5 scaffolds 


\section{Biological characterization Skin irritation}

There was no skin irritation; all the scaffolds had a score of 3 for both edema and erythema. Hence, the scaffolds did not cause any allergic reactions or hypersensitivity reactions.

\section{Antibacterial activity assessment}

For antimicrobial assessment, the prepared scaffolds were tested against six microorganisms, resistant clinical isolates of Staphylococcus aureus 2586, Staphylococcus aureus 2590, Staphylococcus aureus 2583, Staphylococcus aureus 2587, Staphylococcus aureus 2555, and MRD Escherichia coli 1808 using the agar diffusion method to assess their antimicrobial activity. The growth of $E$. coli was not inhibited by either the control drug (erythromycin) or the PKCM1 electrospun keratin/Co Q10/PVA scaffold which did not contain any mupirocin. Electrospun keratin/Co Q10/PVA scaffold containing mupirocin PKCM2, PKCM3, PKCM4, and PKCM5 showed antimicrobial activity against all the clinical isolates of Staphylococcus aureus (Fig. 5a-d). Oxoid ${ }^{\mathrm{Tm}}$ antimicrobial susceptibility disc for erythromycin was seen to have activity against Staphylococcus aureus 2587 and Staphylococcus aureus 2586 . There was no activity against multidrug resistant bacteria with the Oxoid ${ }^{\mathrm{TM}}$ antimicrobial susceptibility disc for the control drugs, compared to the scaffolds which showed some activity.

The strongest activity was observed with PKCM3 with zone of inhibition $18.5 \mathrm{~mm}$ against Staphylococcus aureus 2587. There was a statistically significant difference between all the scaffolds containing mupirocin and control drug with PKCM 2, 3, 4, and 5 having excellent activity against Staphylococcus aureus, hence the fabricated scaffold could be more effective for treatment of infected wounds.

\section{Cytotoxicity and cell proliferation assay}

Biocompatible polymers and their products must be of a less toxic nature with assured cell viability. By ISO 10993-5 standards, anything that reduces cell viability by more than $30 \%$ can be cytotoxic to the membranes. All the electrospun fabricated PKMC scaffolds were found to have viable cell percentage above $100 \%$. The cell line studies on the electrospun scaffolds showed their ability to support the growth of keratinocytes as indicated by higher values for \% viability (Fig. 5a, b). All the electrospun scaffolds showed higher cell viability values than the control at 48 and $72 \mathrm{~h}$, with the CoQ10 containing scaffolds PKCM1: 180\%, 179\%; PKCM 3: 152\%, 247\%; PKCM 4: 136\%, 135\%; and PKCM5: 152\%, 148\%, had higher cell viability values than PKCM2 (130\%), which did not contain CoQ10 (Fig. 6).

\section{Antioxidant activity of the of the PKCM scaffolds using DPPH assay}

Coenzyme Q10 (CoQ10) is an effective antioxidant of the cellular membranes hence its inclusion in the electrospun PKCM scaffold development. There was a concentration-dependent increase in DPPH free radical scavenging with increasing scaffold concentration of CoQ10 (Fig. 7). The percentage DPPH free radical scavenging was not influenced by the concentration of CoQ10 in the scaffolds. PKCM5 exhibited the highest percentage DPPH free radical scavenging activity $65.8 \pm$ 1.02. This result was not statistically significantly different from the values obtained for PKCM1, PKCM3, and PKCM4 as shown in Fig. 7. The DPPH scavenging activity of the scaffolds also showed that the electrospinning did not adversely affect/ diminish the antioxidant activity of the scaffold.

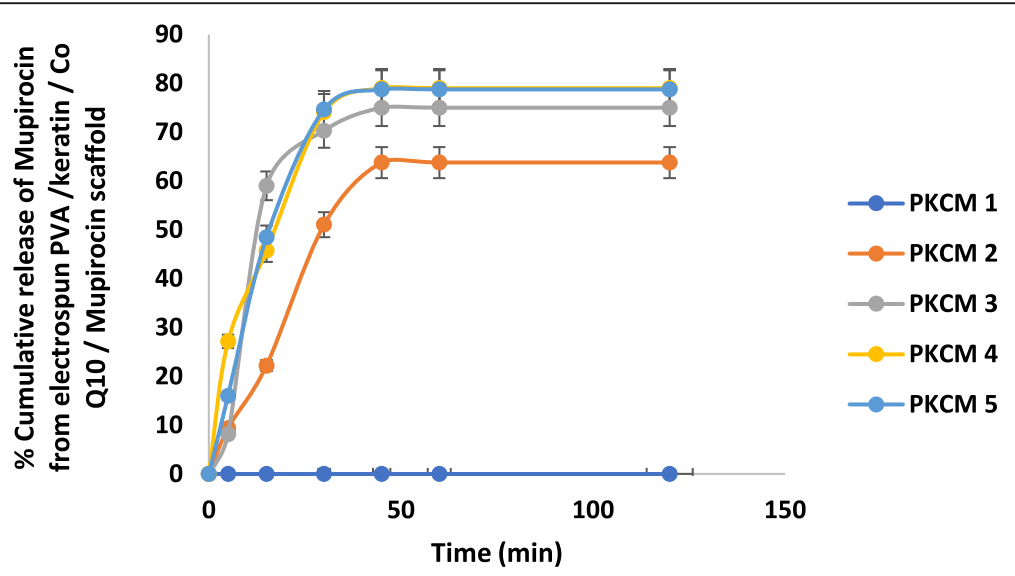

Fig. 4 Cumulative release of mupirocin from electrospun PVA/keratin/Co Q10 mupirocin scaffolds 

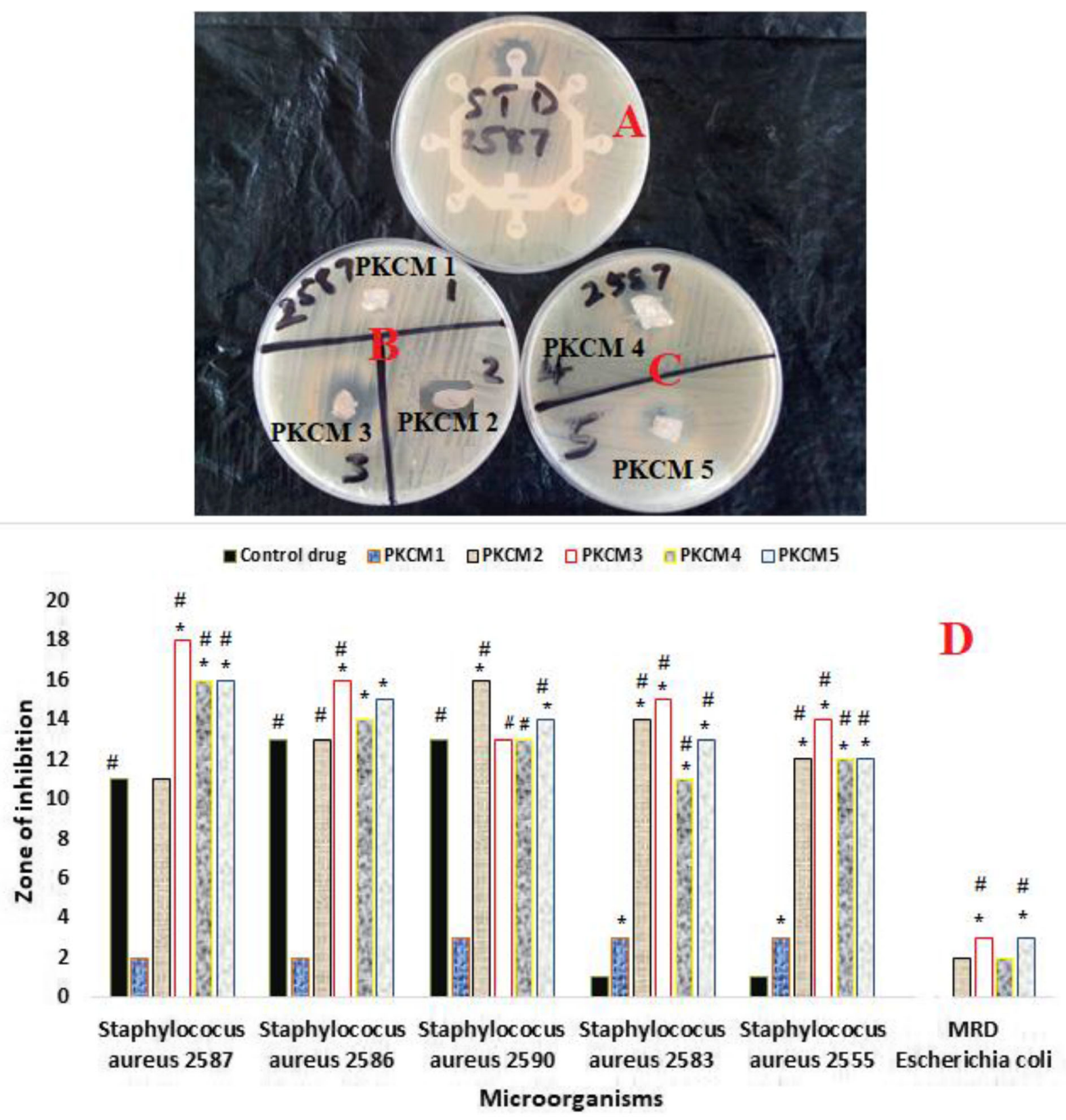

Fig. 5 Evaluation of antibacterial activity of the electrospun keratin/Co Q10/PVA nanofibrous scaffold. a Control drug erythromycin. b Microbial plates showing PKCM1, PKCM2, and PKCM3. c Microbial plates showing PKCM3 and PKCM4. $\mathbf{d}$ Zone of inhibition exhibited by various microorganisms when challenged with control drug and electrospun keratin/Co Q10/PVA nanofibrous scaffolds. ${ }^{*}$ The difference in mean is significant $(P<0.05)$ with respect to control. " The difference in mean is significant $(P<0.05)$ with respect to PKCM1

\section{Discussion}

Electrospun nanofibres have found its place in wound management. Polyvinyl alcohol (PVA) is a popular polymer used because of its biocompatibility and its ability to provide a conducive environment for skin regeneration. This polymer lacks other functional groups and cell recognition sites for functionality at the cellular level is therefore used in combination with bioactive composites like keratin to enhance cell adhesion and faster skin repair $[8,21]$. This new developed electrospun nanofibers combine the biocompatibility potential of PVA, with the bioactive nature of keratin and CoQ10 and the antibacterial property of mupirocin as a new potential for proper wound care.

The presence of CoQ10 in PKCM3, PKCM4, and PKCM5 lowered the surface tension thereby enhancing 


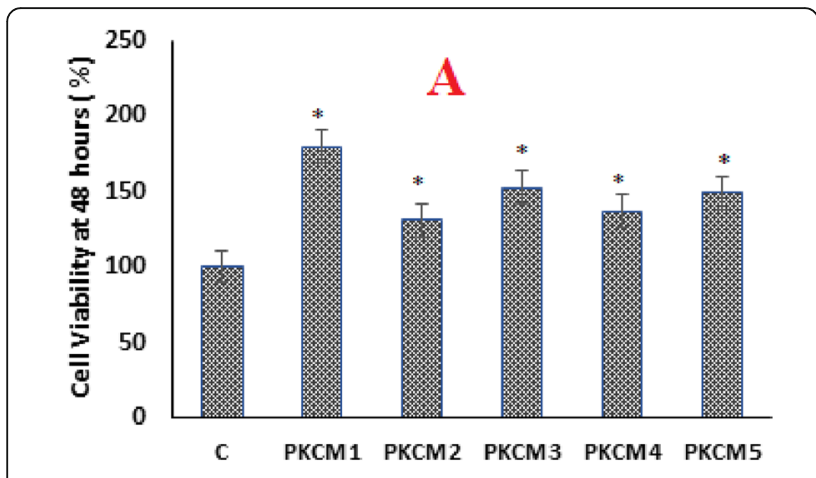

Electrospun PVA /keratin / Co Q10 / Mupirocin scaffold

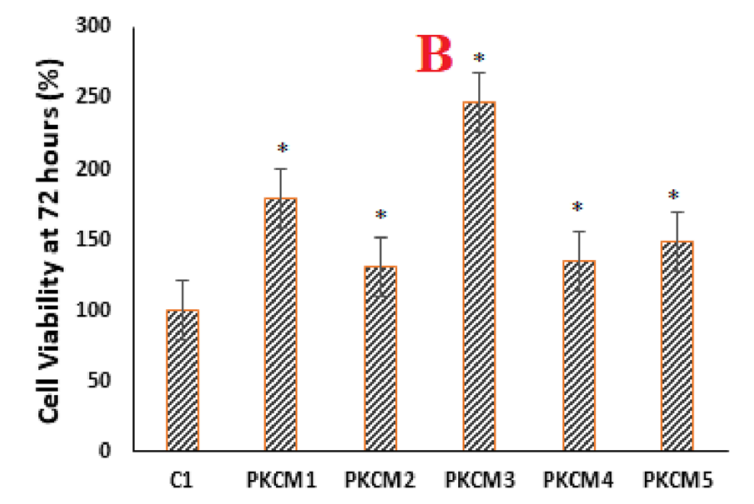

Electrospun PVA /keratin / Co Q10 / Mupirocin scaffold

Fig. $\mathbf{6}$ a Forth-eight hours and $\mathbf{b} 72$ hours cell viability of the control and the electrospun. Treatments were compared with control ${ }^{*} P<0.05$

the morphological features of the fibers. No crystallization of drugs was detected on the polymer surface of the electrospun scaffolds and this suggests that there was a homogeneous dispersion of mupirocin in the electrospun fibers [22-24]. It is desirable that a wound healing material could prevent dehydration of the wound, but also protect it from excess hydration which may impede wound healing. Normal skin has moisture vapor transmission rate (MVTR) of $700-1200 \mathrm{~g} / \mathrm{m}^{2}$ [19], an observed MVTR of in the range $705-750 \mathrm{~g} / \mathrm{m}^{2}$. Twenty-four hours for that samples under study is indicative of the potential of the scaffolds to provide an optimal moist environment locally and hence prevent excessive dehydration while allowing controlled evaporation of wound exudates to promote wound healing. The mechanical studies are carried out to check the strength of the synthesized electrospun scaffolds, which is essential for its biomedical application. The most important criterion for such application is that the samples should be strong enough to avoid the damage. A wound dressing scaffold should have enough mechanical strength to withstand handling both during packaging and wound dressing application. Figure 1a shows the highest mechanical load the electrospun scaffolds can withstand prior to fracture under tension. The scaffolds showed different UTS indicating variations in strengthening effects imparted by the crosslinking agent, ethylene glycol diglycidylether (EDGE), used in this study. The PKCM4 scaffold had the highest UTS at maximum magnitude of $0.2 \mathrm{MPa}$. This could be attributed to promoted wettability and better matrix/fiber adhesion at the concentrations of the various components used in the formulation of PKCM4 scaffold. It has been reported that effective stress transfer between matrix and fiber occurs in composites of enhanced wettability [25]. PKCM5 (UTS of $0.15 \mathrm{MPa}$ ) is the second strongest electrospun composite after PKCM4. Decline in UTS could be attributed to agglomeration and sediment effects associated with filler contents which may ultimately lead to poor wetting between the fillers and matrix; this, according to Pan et al. [26], may result in formation of micro-cracks. PKCM2 possesses the least UTS of $0.03 \mathrm{MPa}$; since PKCM2 is the only formulation without CoQ10, it suggests that the presence of CoQ10 increased the wettability. The native skin has tensile strength and ductility of 5-30 $\mathrm{MPa}$ and $35-115 \%$

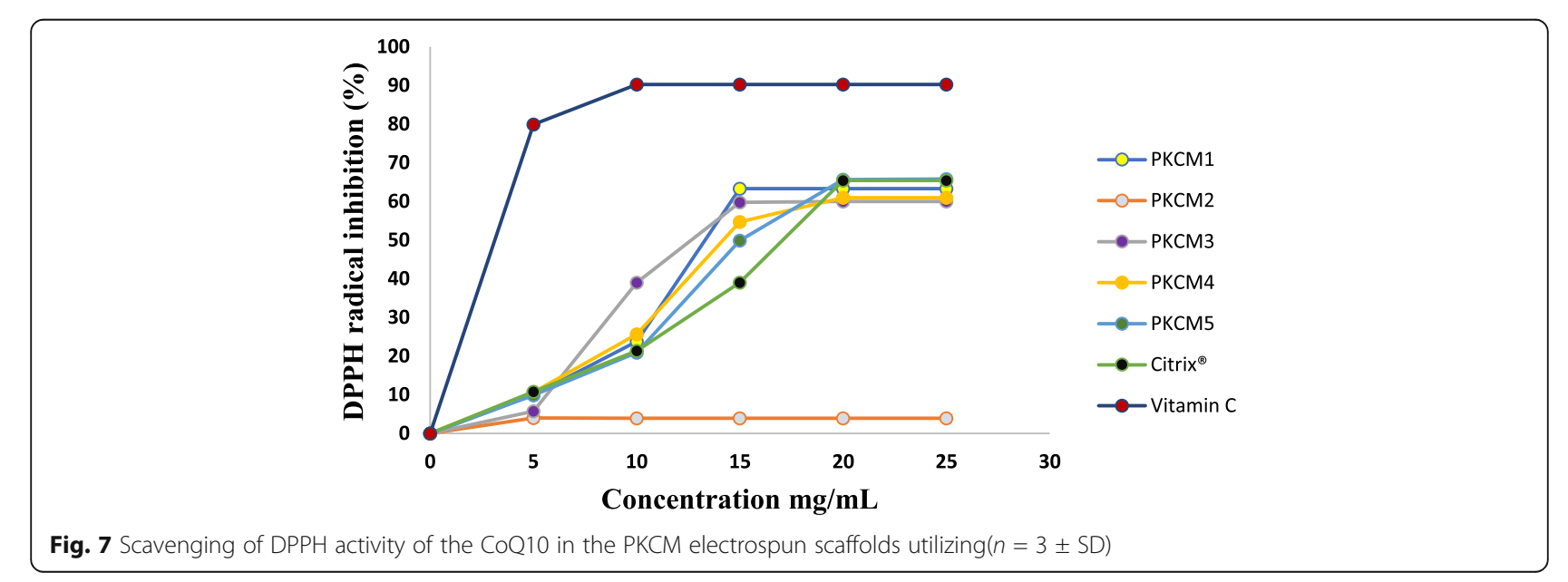


respectively [5]. Though the electrospun scaffolds all have ductility values that fall within the range of the values for the native skin, the corresponding mechanical strengths are below the range. The best capacity to deform before breakage was exhibited by PKCM3, with a ductility of $81.2 \%$. When combined with the value obtained for UTS, PKCM3 possessed adequate mechanical strength and a low enough tensile strength $(0.1 \mathrm{MPa})$ to act as a wound dressing [5]. It has however been reported that scaffolds with low tensile strength offer more advantages for cell culture because they have closer semblance with the collagen, elastin and other soft tissues [5]. Furthermore, the challenge that low tensile strength can pose can be overcome by either using them as primary dressings in direct contact with the wound or by electrospinning them directly on the wound site, using a handheld electrospinning device $[4,26,27]$. PKCM3 showed the highest values in \% porosity and moisture vapor transmission rate, hence optimizing it ability to deter wound bed dehydration while controlling wound exudate flow thus keeping the wound area oxygenated and protected from reinfection [19].

Mupirocin is an effective antibacterial agent and is often used in clinical practice as topical ointment to treat a wide variety of topical wounds. Excellent activity against Staphylococcus aureus, by the fabricated scaffold containing mupirocin, will be effective for treatment of infected wounds. PKCM5 showed the highest cumulative drug release while PKCM2 had the lowest (Fig. 4). PKCM5 contains CoQ10 0.15\%, while there is no CoQ10 in PKCM2. The improved drug release may be attributed to the lowering of the surface tension and improved wettability of the PKCM5 scaffold due to the presence of CoQ10. PKCM3 and PKCM4 contain 0.05\% and $0.1 \%$ of CoQ10 respectively and showed similar drug release patterns. CoQ10 can enhance mitochondrial activity by virtue of being a cofactor in the complexes I-III in the mitochondrial electron transport chain $[10,28]$. The higher cell viability values shown by the CoQ10 electrospun scaffolds can be attributed to increase in cellular energy available for cell growth stimulated by the presence of CoQ10. Topically applied Co Q10 has been shown to have beneficial effects on mitochondrial membrane potential of UV-stressed keratinocytes, suggesting that topically applied ubiquinone reaches the vital layers of the skin and promotes energy metabolism thereby being transformed into ubiquinol [10]. Increase in the antioxidant content of the scaffolds was also attributed to the presence of CoQ10 in the fibers. This is in consonance with Bardania et al. [29], who studied the antioxidant and antimicrobial activity of silver ion nanoparticles for wound healing applications [29-31]. The DPPH scavenging activity of CoQ10 within the scaffolds was not affected by electrospinning process and did not affect the antioxidant activity of the scaffolds; hence, the presence of CoQ10 offered protection to the cells due to the antioxidant properties [30], leading to growth of the keratinocytes used in the study.

\section{Conclusion}

Electrospun nanofibers combining the biocompatibility potential of PVA, with the bioactive nature of keratin and $\mathrm{CoQ} 10$ and the antibacterial property of mupirocin as a new potential for proper wound care, were successfully developed. The PKCM3 scaffolds displayed optimum porosity and ductility and MVTR, important for absorbing excess exudates and maintaining a moist environment on the wound surface. Electrospun keratin/ Co Q10/PVA scaffold containing mupirocin PKCM3 showed excellent antimicrobial activity against all the clinical isolates of 2586, Staphylococcus aureus 2590, 2583, 2587, 2555. The cell line studies on the PKCM3 showed their ability to support the growth of keratinocytes hence the potential of developed scaffolds as a wound dressing. In vivo studies to further investigate the applications of the electrospun keratin/Co Q10/PVA nanofibrous scaffold as a wound dressing is however required.

\section{Abbreviations}

PKCM: Polyvinyl alcohol (PVA) and keratin / Co Q10 fibrous scaffolds; ECM: Extra-cellular matrix; MVTR: Moisture vapor transmission rate; EDGE: Ethylene glycol diglycidylether

\section{Acknowledgement}

The content is solely the responsibility of the authors. The authors acknowledge the technical effort of Mr Olaleye from the Department of Metallurgical and Materials Engineering, Faculty of Engineering, University of Lagos during the electrospinning procedure.

\section{Authors' contributions}

$\mathrm{MOI}$ conceived the study. $\mathrm{MOI}$ and JNA and helped design and coordinate the study. JNA, YAO, and SOA carried out the experimental studies. MOI, CPA, and $\mathrm{Cl}$ drafted the manuscript. All authors have read and approved the final manuscript.

\section{Funding}

Not applicable.

Availability of data and materials

All data and material are available upon request.

Ethics approval and consent to participate

Ethical approval was obtained from the Health Research and Ethics committee of the College of Medicine University of Lagos. All the experiments accorded with the Institution Guidelines and were approved by College of Medicine University of Lagos Health Research Ethical Committee CMUL/HREC/09/19/607.

Consent for publication

Not applicable.

\section{Competing interests}

The authors declare that they have no competing interests.

\section{Author details}

'Department of Pharmaceutics and Pharmaceutical Technology, Faculty of Pharmacy, University of Lagos, PMB 12003, Surulere, Lagos, Nigeria.

${ }^{2}$ Population Council, Center for Biomedical Research, Rockefeller University, New York 10065, USA. ${ }^{3}$ Department of Pharmaceutics and Pharmaceutical 
Technology, Faculty of Pharmacy, Kwame Nkrumah University of Science and Technology, Kumasi, Ghana. ${ }^{4}$ Department of Metallurgical and Materials Engineering, Faculty of Engineering, University of Lagos, PMB 12003, Surulere, Lagos, Nigeria.

Received: 17 March 2020 Accepted: 1 June 2020

Published online: 22 June 2020

\section{References}

1. Frykberg RG, Banks I (2015) Challenges in the treatment of chronic wounds. Advances in Wound Care 4(9):560-582 https://doi.org/10.1089/wound.2015.0635

2. Han G, Ceilley R. (2017) Chronic wound healing: a review of current management and treatments. Advances in Therapy. Springer Healthcare. 34, 599-610 https://doi.org/10.1007/s12325-017-0478-y.

3. Ilomuanya MO, Adeyinka O, Aghaizu C, Cardoso-Daodu I, Akhimien T, Ajayi Tet al., (2019) Co-formulation and characterization of gentamicin-loaded alkyl acrylate cross polymer hydrogel infused with ethanol extract of Tetracarpidium conophorum impregnated on gauze sponge for wound dressing Wound Healing Southern Africa 12(1):22-28 https://journals.co.za/ content/journal/10520/EJC-17b07e7ea5

4. Dong $Y$, Zheng $Y$, Zhang K et al (2020) Electrospun nanofibrous materials for wound healing. Adv. Fiber Mater. https://doi.org/10.1007/s42765-02000034-y

5. Qiang Z, Du Q, Zhao Y, Chen F, Wang Z, Zhang Y, Ni H, Deng H, Li Y, Chen Y (2017) Graphene oxide-modified electrospun polyvinyl alcohol nanofibrous scaffolds with potential as skin wound dressings. RSC Advances 7(46):28826-28836 https://doi.org/10.1039/c7ra03997b

6. Yanfang W, Li P, Xiang P, Lu J, Yuan J, Shen J (2016) Electrospun polyurethane/keratin/AgNP bio composite mats for biocompatible and antibacterial wound dressings. J. Mater. Chem. B 4:635 https://doi.org/10. 1039/C5TB02358K

7. Marek K, Sulejczak D, Czuwara J, Kosson P, Misicka A, Lipkowski AW, Rudnicka $L$ (2017) The role of allogenic keratin-derived dressing in wound healing in a mouse model. Wound Repair Regen 25(1):62-74 https://doi. org/10.1111/wrr.12500

8. Kelly R (2017) Keratins in wound healing. In: Wound Healing Biomaterials, vol 2. Elsevier Inc, pp 353-365 https://doi.org/10.1016/B978-1-78242-456-7. $00017-9$

9. Poovelikunnel TG, Gethin G, Humphreys H (2015) Mupirocin resistance: clinical implications and potential alternatives for the eradication of MRSA.J Antimicrob Chemother 70(10):2681-2692 https://doi.org/10.1093/jac/dkv169

10. Zhigang M, Wu JH, Dong T, Wu MX (2016) Additive enhancement of wound healing in diabetic mice by low level light and topical CoQ10. Scientific Reports 6 https://doi.org/10.1038/srep20084

11. Anja K, Achterberg V, Smuda C, Mielke H, Sperling G, Dunckelmann K, Vogelsang A et al (2015) Topical treatment with coenzyme Q10-containing formulas improves skin's Q10 level and provides antioxidative effects. BioFactors 41(6):383-390 https://doi.org/10.1002/biof.1239

12. Waghmare VS, Wadke PR, Dyawanapelly S, Deshpande A, Jain R, Dandekar P (2018) Starch based nanofibrous scaffolds for wound healing applications. Bioactive Materials 3(3):255-266 https://doi.org/10.1016/j.bioactmat.2017.11.006

13. Zheng L, Li H, Yu H, Kang G, Xu T, Yu J, Li X, Xu H (2018) Modified liquidliquid displacement porometry and its applications in Pd-based composite membranes. Membranes, 2018; 8(2), 29. https://doi.org/10.3390/ membranes8020029

14. David SR, Malek N, Mahadi AH, Chakravarthi S, Rajabalaya R (2018) Development of controlled release silicone adhesive-based mupirocin patch demonstrates antibacterial activity on live rat skin against Staphylococcus aureus. Drug Des Devel Ther 12:481-494 https://doi.org/10.2147/DDDT. S146549

15. National Institutes of Health guide for the care and use of laboratory animals (NIH Publication No. 8023, revised in 1978) https:/grants.nih.gov/ grants/olaw/guide-for-the-care-and-use-of-laboratory-animals.pdf

16. Kilkenny C, Browne W, Cuthill IC, Emerson M, Altman DG (2010) NC3Rs Reporting guidelines working group. Animal research: reporting in vivo experiments: the ARRIVE guidelines. Br J Pharmacol 160(7):1577-1579 https://doi.org/10.1111/j.1476-5381.2010.00872.x

17. Ilomuanya MO, Akhimien T, Aghaizu C, Adeyinka O, Ajayi T (2018) Polyherbal antioxidant topical preparation comprising ethanol extract of Tetracarpidium conophorum and Ocimum gratissimum: formulation and evaluation. Dhaka Univ. J. Pharm. Sci. 17(2):213-219 https://doi.org/10.3329/ dujps.v17i2.39178

18. Balaji S, Kumar R, Sripriya R, Kakkar P, Ramesh DV, Reddy PNK, Sehgal PK (2012) Preparation and comparative characterization of keratin-chitosan and keratin-gelatin composite scaffolds for tissue engineering applications. Mater Sci Eng C 32(4):975-982 https:/::doi.org/10.1016/j.msec.2012.02.023

19. Xu R, Xia H, He W, Li Z, Zhao J, Liu B, Wang Y, Lei Q, Kong Y, Bai Y, Yao Z, Yan R, Li H, Zhan R, Yang S, Luo G, Wu J (2016) Controlled water vapor transmission rate promotes wound healing via wound re-epithelialization and contraction enhancement. Scientific reports 6:24596 https://doi.org/10. 1038/srep24596

20. Kong J, Yu S (2007) Fourier transform infrared spectroscopic analysis of protein secondary structures. Acta Biochim Biophys Sin 39(8):549-559 https://doi.org/10.1111/j.1745-7270.2007.00320.x

21. Hill P, Brantley H, Van Dyke M (2010) Some properties of keratin biomaterials: Kerateines. Biomaterials 31(4):585-593 https://doi.org/10.1016/j. biomaterials.2009.09.076

22. Minoo S, Arab-Sorkhi S, Vatani H, Bagheri-Pebdeni A (2015) New wound dressing polymeric nanofiber containing green tea extract prepared by electrospinning method. Fibers and Polymers 16(8):1742-1750 https://doi. org/10.1007/s12221-015-5297-7

23. Sill TJ, von Recum AH (2008) Electrospinning: applications in drug delivery and tissue engineering. Biomaterials 29(13):1989-2006 https://doi.org/10. 1016/j.biomaterials.2008.01.011

24. Jiajia X, Wu T, Dai Y, Xia Y (2019) Electrospinning and electrospun nanofibers: methods, materials, and applications. Chem Rev 119(8):52985415 https://doi.org/10.1021/acs.chemrev.8b00593

25. Zhaogian L, Xiaodong Z, Chonghua P (2011) Effect of sisal fiber surface treatment on proper-ties of sisal fiber reinforced polylactide composites Int. J. Polym. Sci Article ID 803428:7 https://doi.org/10.1155/2011/803428

26. Pan MZ, Zhou DG, Bousmina M, Zhang SY (2009) Effects of wheat straw fiber content and characteristics and coupling agent concentration on the mechanical properties of wheat straw fiber-polypropylene composites. J. Appl. Polym. Sci. 113:1000-1007. https://doi.org/10.1002/app.29789

27. Ilomuanya MO, Adebona AC, Wang W, Sowemimo AA, Eziegbo C, Silva BO, Adeosun SO, Joubert E, De Beer D (2020) Development and characterization of collagen-based electrospun scaffolds containing silver sulphadiazine and Aspalathus linearis extract for potential wound healing applications. SN Applied Sciences 2:881 https://doi.org/10.1007/s42452-020-2701-8

28. Zaki NM (2016) Strategies for oral delivery and mitochondrial targeting of CoQ10. Drug Delivery 23(6):1868-1881. https://doi.org/10.3109/10717544. 2014.993747

29. Bardania H, Mahmoudi R, Bagheri $\mathrm{H}$ et al (2020) Facile preparation of a novel biogenic silver-loaded Nanofilm with intrinsic anti-bacterial and oxidant scavenging activities for wound healing. Sci Rep 10:6129 https://doi. org/10.1038/s41598-020-63032-5

30. Lagares MA, da Silva GC, Cortes SF, Luz SB, de Resende AC, Alves NC, Wenceslau RR, Stahlberg R (2020) Does coenzyme Q10 exert antioxidant effect on frozen equine sperm? J Equine Vet Sci 88:102964 https://doi.org/ 10.1016/j.jevs.2020.102964

31. Li Z, Zhou X, Pei C. (2011) Effect of sisal fiber surface treatment on properties of sisal fiber reinforced polylactide composites. International Journal of Polymer Science Article ID 803428 https://doi.org/10.1155/2011/ 803428

\section{Publisher's Note}

Springer Nature remains neutral with regard to jurisdictional claims in published maps and institutional affiliations. 